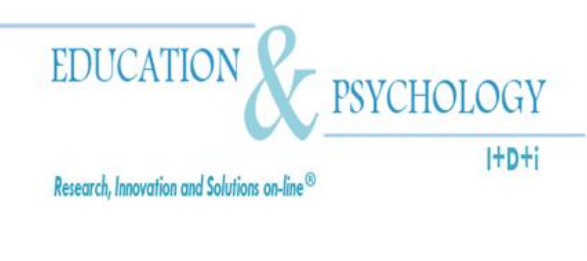

\title{
Electronic Journal of Research
}

in Educational Psychology

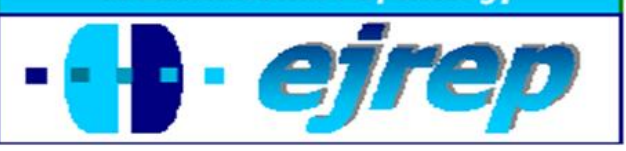

Editorial EOS

\section{Efectos de un programa para desarrollar las habilidades creativas}

\section{Natalia Larraz Rábanos ${ }^{1}$, Pedro Allueva Torres ${ }^{2}$}

${ }^{1}$ Departamento de Ciencias de la Educación, Universidad de Zaragoza, Zaragoza

${ }^{2}$ Departamento de Psicología y Sociología, Universidad de Zaragoza, Zaragoza

\section{España}

Correspondencia: Natalia Larraz Rábanos. Facultad de Educación. Avda. San Juan Bosco, 7, 50009, Zaragoza. España. E-mail: nlarraz@unizar.es 


\section{Resumen}

Introducción. El objetivo de este estudio es presentar los resultados obtenidos de un programa de intervención para el desarrollo de las habilidades creativas en un grupo de estudiantes de segundo curso de Educación Secundaria Obligatoria (ESO).

Método. El programa se ha implementado en un colegio de la ciudad de Zaragoza (España) durante el curso académico 2008-09. El estudio ha seguido un diseño cuasi-experimental de medidas repetidas con grupo control no equivalente. La muestra del estudio estuvo compuesta por 48 participantes de entre 13-14 años (24 del grupo experimental y 24 del grupo de control) mediante un tipo de muestreo "de oportunidad o comodidad". Antes y después del programa se administraron tres pruebas: el Test de Torrance de Pensamiento Creativo (TTCT) como prueba inicial y final y; la Prueba de la Imaginación Creativa para Jóvenes (PIC-J) como prueba de seguimiento. El programa consistió en una serie de sesiones semanales de 50 minutos estructuradas por sus objetivos, actividades y criterios de evaluación.

Resultados. Los resultados del programa, evaluados a través del ANOVA, mostraron que el programa logró un desarrollo significativo $(\mathrm{p}<.05)$ de la creatividad verbal y figurativa del grupo experimental en comparación con el grupo control. Los resultados del estudio muestran un mayor desarrollo de la creatividad verbal que la creatividad figurativa.

Conclusión. Con esta experiencia se demuestra la posibilidad de desarrollar la creatividad en la ESO. Las conclusiones del estudio se centran en resaltar la importancia de integrar el desarrollo de habilidades creativas a través de los objetivos y el proceso de enseñanza-aprendizaje implicado en la metodología educativa del currículum y resaltar la importancia del entrenamiento del profesorado en el desarrollo de las habilidades de pensamiento creativo.

Palabras Clave: Creatividad; Pensamiento creativo, Procesos de enseñanza-aprendizaje, Metodología educativa; Programa de intervención; Educación Secundaria Obligatoria. 


\begin{abstract}
Introduction. The aim of the present study is to present an intervention program for the development of creative skills of a group of students of lower Secondary Education

Method. This program has evolved into a school of Zaragoza (Spain) during the 2008-09 academic year. The study used a repeated measures quasi-experimental design with nonequivalent control group. The sample included 48 participants aged 13-14 years (24 experimental and 24 control participants). Before and after administering the program it was applied three assessments test; the Torrance Test of Creative Thinking (TTCT) was applied as a pretest and postest in their parallel forms, and the Test of Creative Imagination for Young Children (PIC-J) was applied as a follow-up test to improve the reliability of the intervention. The program consisted of weekly 50-minute structured sessions thought their objectives, activities and evaluation criteria.
\end{abstract}

Results. The results of the study, probed by the ANOVA, showed that the program significantly increased $(\mathrm{p}<.05)$ the verbal and the graphic creativity in the experimental group compared with the control group. The verbal creativity development was higher than the graphic creativity development.

Conclusion: The results of this study shows that in order to improve the educational effects of creative skill development is suggested to incorporate the creative development into the objectives through the teaching and learning process of the educational methodology. This aspect highlights the importance of the trainee teacher on creative thinking skills.

Keywords: Creativity; Creative Thinking; Teaching-learning process; Educational methodology; Intervention program; Secondary Education. 


\section{Introducción}

Hoy en día, el desarrollo de las habilidades creativas es fundamental para lograr un aprendizaje de alto nivel y una sociedad más avanzada y se hace necesario incorporarlas a través de los objetivos y de los procesos de enseñanza-aprendizaje del sistema educativo. El inicio del estudio de la creatividad en el ámbito de la psicología comienza con la figura de Guilford (1950). Asimismo numerosos autores han destacado la importancia de la creatividad como una habilidad mental necesaria para el presente y para el futuro de nuestra sociedad (Gardner, 1993, 2006; Sternberg y Lubart, 1995; Torre, 2006; Sampascual, 2007) y, hoy en día, se hacen indudables los beneficios de su desarrollo a cualquier edad y en cualquier etapa educativa.

En general, la creatividad se ha definido como una habilidad para generar ideas y productos nuevos, originales y valiosos. Tradicionalmente la educación formal ha fomentado un tipo de pensamiento convergente, crítico, racional, analítico y secuencial, basado en la reproducción del conocimiento relegando a un segundo plano el desarrollo de la creatividad (De Bono, 1970; Nickerson, Perkins y Smith, 1985; Mayer, 1992; Torre, 2006; Runco, 2007; Sampascual, 2007; Bernabeu y Goldstein, 2009). Sin embargo, a raíz de los estudios realizados en el ámbito de la psicología de la instrucción, se ha demostrado que la creatividad es fundamental para lograr una educación de alto nivel y un aprendizaje exitoso (Sternberg, 1985; Lipman, 1991). Por lo tanto, hoy en día se hace necesario desarrollar el pensamiento creativo, ya que es fundamental para lograr un aprendizaje de alto nivel y está insuficientemente desarrollado en el sistema educativo.

La importancia de desarrollar la creatividad en las distintas etapas educativas está patente a través de las diferentes acciones, programas e iniciativas existentes a nivel internacional, europeo y nacional. La mayoría de estos programas y proyectos muestran un desarrollo positivo de la creatividad tras su intervención. A nivel internacional destacamos, entre otros, el Programa de Pensamiento Productivo de Covington, Crutchfield y Davis (1966), el Programa CoRT de De Bono (1973, 1985), el programa de De Sánchez (1996) y la iniciativa de Perkins (1993). A su vez, el Proyecto Cero (Harvard University, 2010) ideado inicialmente por Goodman a finales de los años 60 , tiene como uno de sus principales objetivos el desarro- 
llo de la creatividad y cuenta con numerosos programas que han sido aplicados endistintos continentes y países.

A nivel europeo, destacamos diferentes estudios como el Método de Aprendizaje Creativo (European Union, 2005), el Proyecto de Creatividad para la Educación Superior (European Universtity Association, 2007) y el proyecto de Comunidades de Aprendizaje Creativo (Education, Audiovisual \& Culture Executive Agency, 2008; European Union, 2010). Todos estos proyectos han surgido como iniciativas aplicadas en diferentes niveles y etapas educativas, como respuesta al Año Europeo de la Creatividad y la Innovación 2009, propuesto por la Unión Europea (European Union, 2008). Hoy en día, dichas iniciativas siguen vigentes debido a la inclusión de la creatividad como una de las habilidades centrales para lograr alcanzar las competencias clave para el Aprendizaje Permanente (European Union, 2006).

Por último, a nivel nacional destacamos los trabajos desarrollados por López (2001) que diseña y evalúa un programa para el desarrollo de la creatividad en estudiantes de Educación Infantil y Primaria. También resaltamos el estudio de Prieto, López, Bermejo, Renzulli y Castejón (2002) basado en la aplicación y evaluación del programa de intervención de Renzulli y colaboradores para el desarrollo de la creatividad en estudiantes de niveles de Educación Infantil y primer ciclo de Educación Primaria. Además, Navarro (2008) aplica el mismo programa de intervención de Renzulli y colaboradores en estudiantes de Educación Primaria. En último lugar, cabe señalar el programa desarrollado y aplicado por Garaigordobil y Berrueco (2011) para desarrollar la creatividad a través del juego en estudiantes de Educación Infantil con la obtención de resultados positivos en el desarrollo de la creatividad tras su intervención.

El desarrollo de la creatividad es importante en todas las etapas educativas pero su desarrollo se considera especialmente importante en la etapa de la Educación Secundaria Obligatoria (ESO) ya que este periodo educativo va a ser fundamental para el desarrollo cognitivo, personal y social de los estudiantes. En esta etapa del desarrollo se va a elaborar el Proyecto de Vida, un tipo de pensamiento superior y un tipo de racionalidad más avanzada. En este sentido, la creatividad va a influir de forma positiva para lograr dichos aspectos ya que, el pensamiento adulto es más parecido a un tipo de pensamiento posformal (Arlin, 1975), descrito como un quinto estadio del desarrollo cognitivo caracterizado por ser eminentemente creativo, lo que va a propiciar un mayor y mejor desarrollo cognitivo, una mayor capacidad de 
elección en las decisiones futuras, en función de unos intereses y criterios más amplios. Por otro lado, se han encontrado muestras de que los programas de esta etapa educativa no incluyen una metodología que fomente la creatividad de forma suficiente, lo que puede llevar a la desmotivación de nuestros estudiantes y su consiguiente fracaso escolar (Woolfolk, 2010).

\section{Objetivos e hipótesis}

Según los antecedentes expuestos, el estudio propuesto tiene como objetivo diseñar, aplicar y evaluar los efectos de un programa de intervención para el desarrollo de las habilidades creativas de un grupo de estudiantes de segundo curso de ESO. Dicho programa se fundamenta en el modelo de la Estructura del Intelecto de Guilford (1967a, 1967b) y persigue desarrollar los procesos de producción divergente propuestos por el mismo autor (fluidez, flexibilidad, originalidad y elaboración). Además de estos procesos de producción divergente, se han fomentado otros procesos de pensamiento que sirven para estimular la creatividad, tales como el pensamiento crítico y el pensamiento metacognitivo aplicados a la solución de problemas (Allueva, 2007; Larraz, 2011). En este sentido, se puede afirmar que distintos autores (Nickerson, Perkins y Smith, 1985; Sternberg, 1985; Lipman, 1991) apoyan la idea de que las habilidades del pensamiento de orden superior implicadas en la solución de problemas son las que más directamente relacionadas están con el aprendizaje de alto nivel y dichas habilidades están fundamentalmente compuestas por las habilidades del pensamiento crítico, creativo y metacognitivo.

El programa diseñado se ha aplicado con la finalidad de desarrollar las habilidades creativas del grupo al que se dirige, como población diana y se ha evaluado con la finalidad de probar sus efectos y proponer una metodología adecuada para ello, ya que en esta edad, las habilidades creativas están insuficientemente atendidas y desarrolladas a través de los procesos de enseñanza-aprendizaje, tal y como comentábamos con anterioridad. Dicho programa consiste en un conjunto de sesiones estructuradas para que el profesorado, debidamente instruido en la metodología del mismo, estimule el potencial creativo de los estudiantes mediante la adquisición y aplicación de una serie de estrategias creativas y mediante la utilización y aplicación de los procesos de pensamiento divergente aplicados a la solución de problemas. Para ello se considera esencial, en primer lugar, crear un ambiente favorable, por lo que se hace necesario eliminar las barreras que limitan el pensamiento creativo (Osborn, 1953; Simberg, 1971; Lorna, 1998; Recio, 2005), que pueden provenir de uno mismo como del contexto (escuela, familia, sociedad). 
Por lo tanto, para fomentar la creatividad de forma global se sugiere estimular un estilo de pensamiento creativo a través de la aplicación de los procesos de pensamiento divergente implicados en la solución de problemas mediante el uso de distintas estrategias con la finalidad de generar productos creativos.

\section{Método}

\section{Participantes}

La población a la que se destina el programa de intervención son los estudiantes españoles de ESO. El programa se ha adaptado a las características psicoeducativas de la población destinataria y a su variabilidad, teniendo en cuenta las diferencias individuales de cada estudiante. Los participantes del estudio han sido tratados conforme a los principios éticos internacionales para la investigación científica.

En este estudio se han tomado dos grupos-clase de estudiantes de segundo curso de ESO (Nivel 2 de Educación Secundaria según la Clasificación Internacional Normalizada de la Educación) (UNESCO, 2011) de un centro de Educación Infantil, Primaria y Secundaria de la ciudad de Zaragoza. La selección de la muestra se ha realizado mediante un tipo de muestreo denominado "de oportunidad o de comodidad" (Coolican, 2009), en el que se utilizan los grupos naturales existentes en cada grupo-clase como procedimiento para configurar los grupos y asignarlos a las condiciones de tratamiento y de control de forma aleatoria.

El tamaño de la muestra del estudio es de $\mathrm{N}=48$ participantes de ambos géneros ( $48 \%$ de mujeres y $52 \%$ de varones), de los cuales, la mitad $(n=24)$ forman parte del grupo experimental (GE en adelante) (que se ha visto reducido a $n=21$ debido a la mortalidad experimental a lo largo de la intervención, para ello se ha tenido en cuenta la asistencia a un mínimo del $90 \%$ de las sesiones, los datos recogidos de los participantes que no cumplían con este criterio se han desestimado), y la otra mitad $(n=24)$ del grupo de control (GC en adelante). Por tanto, los grupos están equiparados en cuanto al número de participantes presentes en cada condición del estudio (47\% en la condición de tratamiento -GE- y 53\% en la condición de no tratamiento- GC-). La edad media del GE es de 13’2 años ( $\mathrm{SD}=0$ ’58) y la edad media del GC es de 13'17 años ( $\mathrm{SD}=0$ ’38). 


\section{Instrumentos}

Los instrumentos de evaluación de las habilidades creativas son el Test de Torrance de Pensamiento Creativo (TTCT - Torrance Test of Creativity Thinking) (Torrance, 1966) y la Prueba de Imaginación Creativa para Jóvenes, PIC-J (Artola, et al., 2008).

El TCTT se ha utilizado en su Forma A para la evaluación inicial y su Forma B para la evaluación final. El TTCT es una prueba utilizada para evaluar los procesos de producción divergente propuestos por Guilford (fluidez, flexibilidad, originalidad y elaboración) en niños y adolescentes. La fluidez evalúa el número de ideas emitidas, la flexibilidad evalúa la variedad de las ideas emitidas en función del número de categorías existentes, la originalidad evalúa la novedad de las ideas emitidas en función de su infrecuencia y la elaboración evalúa los detalles que mejoran la calidad de las ideas emitidas. El TTCT consta de dos subpruebas, que evalúan dos formas principales de la creatividad: la creatividad verbal y la creatividad figurativa. Esta prueba se ha escogido por ser un referente a nivel internacional para la evaluación de la creatividad, aún vigente por su fiabilidad y validez, contrastada en diversos estudios (Ferrando, Ferrándiz, Bermejo, Sánchez, Parra y Prieto, 2007; Runco, 2010).

La PIC-J se ha utilizado para realizar la evaluación de seguimiento de los efectos del programa. Esta prueba consta de cuatro subtest, tres de ellos evalúan la creatividad narrativa (verbal en el TCTT) y uno de ellos la creatividad gráfica (figurativa en el TCTT) y es similar por sus características al TTCT. Se trata de un instrumento estandarizado que ha sido validado con población española y evalúa la fluidez, la flexibilidad, la originalidad, la elaboración y dos componentes adicionales al TTCT de la creatividad figurativa, que son el título, definido como la "aptitud del sujeto para elaborar una frase adecuada al dibujo, más o menos sorprendente y no meramente descriptiva" (Artola, et al., 2008, p. 29) y los detalles especiales definidos como la "aptitud del sujeto para asignar detalles como uniones, inversiones, expansiones,...a los diferentes dibujos" (Artola, et al., 2008, p. 29). Esta prueba se ha escogido porque ha sido contrastada y evaluada por sus autores a nivel nacional y presenta evidencias de ser una prueba adecuada para evaluar la creatividad, así como unos índices elevados de fiabilidad y validez contrastados por los autores.

\section{Procedimiento}

El procedimiento del estudio consiste en el diseño de un programa de intervención para desarrollar las habilidades creativas de un grupo de estudiantes de segundo curso de ESO. 
La intervención ha seguido un diseño cuasi-experimental con grupo de control no equivalente con medidas antes y después. Además se ha administrado una prueba de seguimiento transcurridos tres meses desde la prueba final con el fin de evaluar el grado de fijación de los efectos del programa y su fiabilidad (ver la Tabla 1).

Tabla 1. Esquema del diseño de la intervención.

\begin{tabular}{cccccc}
\hline \multirow{2}{*}{ Grupo } & \multirow{2}{*}{ Asignación } & \multicolumn{3}{c}{ SECUENCIA DE REGISTRO } \\
\cline { 3 - 5 } & & $\begin{array}{c}\text { Prueba } \\
\text { Inicial }\end{array}$ & Programa & $\begin{array}{c}\text { Prueba } \\
\text { Final }\end{array}$ & $\begin{array}{c}\text { Prueba de } \\
\text { Seguimiento }\end{array}$ \\
\hline Experimental & $\mathrm{NA}$ & $\mathrm{O}_{1}$ & $\mathrm{X}$ & $\mathrm{O}_{2}$ & $\mathrm{O}_{3}$ \\
Control & $\mathrm{NA}$ & $\mathrm{O}_{1}$ & ----- & $\mathrm{O}_{2}$ & $\mathrm{O}_{3}$ \\
\hline
\end{tabular}

Nota. NA: asignación de los participantes a los grupos no conocida ni aleatoria.

El diseño del programa para el desarrollo de las habilidades creativas se ha realizado a través de la creación de una serie de actividades estimulantes y motivadoras adaptadas al nivel de desarrollo de los participantes y su planteamiento se ha contrastado con el profesorado y con el Departamento de Orientación del centro. El programa consta de siete actividades que se han aplicado durante nueve sesiones en las que se ha desarrollado la creatividad así como otros procesos de pensamiento aplicados a la solución de problemas que pueden estimularla y potenciarla, en un ámbito de conocimiento específico (asignatura de Tecnologías) e inespecífico (asignatura de Tutoría). El periodo de aplicación del programa se hizo en el curso académico 2008/09, durante el cual se estableció la toma de contacto con el centro, la instrucción del profesorado que participó en su aplicación y la implementación y la evaluación del programa y tuvo una duración de siete meses desde la prueba inicial hasta la prueba de seguimiento. La instrucción previa al profesorado que imparte el programa siguió los criterios conceptuales y metodológicos establecidos en el mismo. La intervención se realizó en coordinación con el profesorado del centro y con el Departamento de Orientación, de acuerdo al Plan de Acción Tutorial (PAT) del centro.

La estructura del programa ha sido la siguiente:

- Evaluación inicial de la creatividad mediante el Test Pensamiento Creativo de Torrance (Torrance, 1966). Forma A. Se recogen los datos relativos a la evaluación inicial de la creatividad del grupo experimental y control.

- Sesiones del programa en el grupo experimental o de intervención. 
- Evaluación final mediante la aplicación del Test de Pensamiento Creativo (Torrance, 1966). Forma B. Se recogen los datos relativos a la evaluación final de la creatividad del grupo experimental y control.

- Evaluación de seguimiento de la creatividad mediante la PIC-J (Artola, et al., 2008). Se recogen los datos relativos a la evaluación de seguimiento de la creatividad del grupo experimental y control.

Para desarrollar la creatividad durante las distintas sesiones del programa se han tenido en cuenta las siguientes orientaciones: a) Conocer, eliminar y superar las barreras que impiden el pensamiento creativo; b) Fomentar una disposición o actitud positiva a explorar y expresar el potencial creativo para aplicar los procesos de pensamiento creativo y a desarrollar la creatividad en el aula; c) Estimular un estilo de pensamiento creativo; d) Lograr la creación de productos creativos a través de la aplicación del proceso creativo implicado en la solución de problemas; e) Fomentar el uso de los procesos de producción divergente descritos por Guilford (1976) (Fluidez, Flexibilidad, Originalidad y Elaboración) en las distintas tareas, las distintas personas y las distintas estrategias empleadas; f) Considerar los factores sociales que estimulan la creatividad (fomentar la autoeficacia creativa, la motivación intrínseca y la evaluación interna hacia la tarea y reforzar las situaciones creativas); g) Considerar las características individuales de cada estudiante; h) Fomentar el uso, aprendizaje y transferencia de las estrategias de aprendizaje para desarrollar las habilidades creativas.

Las sesiones del programa se han estructurado en torno a unos objetivos, contenidos, metodología, actividades y criterios de evaluación dentro de las materias de aprendizaje de Tecnologías y Tutoría. Las actividades se han basado en la generación de ideas y en la solución de problemas en las que se debe aplicar y utilizar distintas estrategias creativas para desarrollar la creatividad verbal y figurativa tales como:

- Charla informativa sobre el programa y las actividades a realizar, los factores y los aspectos más significativos de la creatividad.

- Breve descripción de los procesos de producción divergente de Guilford (1976). Actividad para conocer y desarrollar la creatividad figurativa a través de completar dibujos mediante la estrategia de la "Tormenta de ideas" (Osborn, 1953). 
- Evaluación y conocimiento de las barreras del pensamiento creativo. Aplicación y corrección del Inventario de las barreras del pensamiento creativo y de la acción innovadora de Lorna (1998).

- Indicar posibilidades para el reciclado de objetos mediante la estrategia de la "Tormenta de ideas" (Osborn, 1953).

- Diseñar objetos mediante la estrategia de la "Lista de comprobación" (Osborn, 1953).

- Inventar una historia mediante acontecimientos narrados e imaginarios a través del uso de la metáfora, la analogía, las relaciones, las invenciones, las fábulas, etc. (Rodari, 2004).

- Conocer y aplicar el proceso creativo en la solución de problemas de Amabile (1983).

Cada sesión ha sido estructurada en relación a unos criterios de evaluación y corrección que se debían aplicar para llevar un seguimiento diario de la evolución y desarrollo de la creatividad en todas ellas. Los datos de cada sesión han sido recogidos en registros narrativos y analizados posteriormente para comparar la evolución de los resultados de los ejercicios en relación a las pruebas realizadas y a la propia evolución del programa de intervención.

\section{Análisis Estadístico}

El análisis estadístico realizado para obtener los resultados en relación al desarrollo de las habilidades creativas antes y después del programa de intervención se ha obtenido a nivel nomotético e ideográfico con el fin de observar la concordancia de los resultados obtenidos en ambos niveles. Para el estudio nomotético de los efectos del programa entre la prueba inicial y final se ha realizado un ANOVA con puntuaciones de cambio intragrupal e intergrupal y en la prueba de seguimiento se ha realizado un ANOVA ordinario intergrupal. Para el estudio ideográfico de los efectos del programa entre la prueba inicial y final, se analiza la proporción de desarrollo de las habilidades creativas de los participantes.

\section{Resultados}

A nivel nomotético, en la Tabla 2 se presentan los resultados del análisis inferencial obtenidos de las diferencias intragrupales e intergrupales debidas a la intervención de cada una de las variables de la de la creatividad verbal y figurativa en ambos grupos de estudio. En la primera columna se presenta la puntuación media de cambio en un mismo grupo (diferencia intragrupal) y la desviación estándar, mientras que en la segunda columna, se presenta la significación de dicha diferencia. En las tres columnas siguientes se presenta la diferencia media 
de cambio entre los dos grupos de estudio (diferencia intergrupal), la desviación estándar, la probabilidad de cometer error tipo I $(\alpha)$ y su significación. El Nivel de Confianza se ha fijado en el $95 \%$.

Tabla 2. Media, desviación estándar y resultados del ANOVA de las diferencias intragrupales e intergrupales en la creatividad debidas al programa de intervención.

\begin{tabular}{|c|c|c|c|c|c|c|c|}
\hline \multirow{2}{*}{ Creatividad Verbal } & \multirow{2}{*}{ Grupo } & \multicolumn{3}{|c|}{ Diferencia Intragrupal } & \multicolumn{2}{|c|}{ Diferencia Intergrupal } & \multirow{2}{*}{$p$} \\
\hline & & $\mathrm{M}$ & SD & & $\mathrm{M}$ & SD & \\
\hline \multirow[t]{2}{*}{ Fluidez } & $\mathrm{E}$ & 17. & 7.6 & $<.05$ & 42. & 10.4 & \multirow[t]{2}{*}{$.001 * * *$} \\
\hline & $\mathrm{C}$ & - & 7.1 & $<.01$ & & & \\
\hline \multirow[t]{2}{*}{ Flexibilidad } & $\mathrm{E}$ & 9.4 & 2.3 & $<.01$ & 11. & 3.2 & \multirow[t]{2}{*}{$.002 * *$} \\
\hline & $\mathrm{C}$ & - & 2.2 & $>.05$ & & & \\
\hline \multirow{2}{*}{ Originalidad } & $\mathrm{E}$ & 32. & 10.3 & $<.05$ & 50. & 14.1 & \multirow{2}{*}{$.001 * *$} \\
\hline & $\mathrm{C}$ & - & 9.6 & $>.05$ & & & \\
\hline \multirow{2}{*}{ Creatividad Verbal Total } & $\mathrm{E}$ & 59. & 19.3 & $<.05$ & 104 & 26.5 & \multirow[t]{2}{*}{$.0001 * * *$} \\
\hline & $\mathrm{C}$ & - & 18.1 & $<.05$ & ح & & \\
\hline \multirow{2}{*}{ Creatividad Figurativa } & \multirow{2}{*}{ Grupo } & \multicolumn{2}{|c|}{ Diferencia Intragrupa } & \multirow{2}{*}{$p$} & \multicolumn{2}{|c|}{ Diferencia Intergrupal } & \multirow{2}{*}{$p$} \\
\hline & & $\mathrm{M}$ & SD & & $\mathrm{M}$ & SD & \\
\hline \multirow{2}{*}{ Fluidez } & $\mathrm{E}$ & 8.1 & 1.9 & $<0.5$ & 1.1 & 2.5 & \multirow{2}{*}{.655} \\
\hline & $\mathrm{C}$ & 6.5 & 1.7 & $<0.5$ & & & \\
\hline \multirow[t]{2}{*}{ Flexibilidad } & $\mathrm{E}$ & 1.8 & 1.0 & $>.05$ & 2.1 & 1.4 & \multirow[t]{2}{*}{.160} \\
\hline & $\mathrm{C}$ & - & 0.9 & $>0.5$ & & & \\
\hline \multirow{2}{*}{ Originalidad } & $\mathrm{E}$ & 14. & 3.3 & $<.05$ & 5.3 & 4.9 & \multirow[t]{2}{*}{.094} \\
\hline & $\mathrm{C}$ & 9.3 & 3.4 & $<.5$ & & & \\
\hline \multirow[t]{2}{*}{ Elaboración } & $\mathrm{E}$ & 19. & 3.8 & $<.05$ & 26. & 5.2 & \multirow[t]{2}{*}{$.0001 * * *$} \\
\hline & $\mathrm{C}$ & - & 3.5 & $<.05$ & & & \\
\hline \multirow{2}{*}{ Creatividad Figurativa Total } & $\mathrm{E}$ & 44. & 8.1 & $<.05$ & 36. & 11.2 & \multirow{2}{*}{$.001 * *$} \\
\hline & $\mathrm{C}$ & 8.1 & 7.6 & $>.05$ & & & \\
\hline
\end{tabular}

Nota. E: Experimental; C: Control; *** $p<.001 . * * p<.01 . * p<.05$.

Según los resultados obtenidos a nivel nomotético, las diferencias observadas intragrupales e intergrupales debidas al programa de intervención son las siguientes (Tabla 2):

- A nivel intragrupal, el desarrollo de la creatividad verbal y figurativa del GE es significativo en todas las variables de la creatividad $(\mathrm{p}<.05)$ excepto en la flexibilidad figurativa $(p>.05)$.

- A nivel intergrupal, el desarrollo de la creatividad del GE es significativamente mayor al desarrollo de la creatividad del GC en todas las variables de la creatividad verbal (fluidez: $p<.01$; flexibilidad: $p<.01$; originalidad: $\mathrm{p}<.01 \mathrm{y}$; creatividad verbal total: $\mathrm{p}<.001)$, en la creatividad figurativa total $(p<0.01)$ y en la elaboración $(p<.001)$. El desarrollo de la fluidez, flexibilidad y originalidad figurativa, es mayor en el GE 
que en el GC, sin embargo estas diferencias no son significativas entre un grupo y otro $(p>.05)$.

En la prueba de seguimiento, las puntuaciones de las pruebas del TCTT y la PIC-J no son comparables entre sí a nivel intragrupal debido a que están en diferente escala de medida. Por este motivo, solamente se analizan las diferencias observadas a nivel intergrupal en un mismo momento de evaluación (Tabla 3).

Tabla 3. Media, desviación estándar y resultados del ANOVA de las diferencias intergrupales en la creatividad de la prueba de seguimiento (PIC-J).

\begin{tabular}{|c|c|c|c|c|}
\hline Variable & Grupo & M & SD & $\mathrm{p}$ \\
\hline \multirow{2}{*}{ Creatividad Narrativa } & $\mathrm{E}$ & 62.5 & 7.0 & \multirow{2}{*}{$.009 * *$} \\
\hline & $\mathrm{C}$ & 36.3 & 6.5 & \\
\hline \multirow{2}{*}{ Fluidez Narrativa } & $\mathrm{E}$ & 54.1 & 7.1 & \multirow{2}{*}{.072} \\
\hline & $\mathrm{C}$ & 36.6 & 6.6 & \\
\hline \multirow{2}{*}{ Flexibilidad Narrativa } & $\mathrm{E}$ & 61.4 & 6.4 & \multirow{2}{*}{$.0001 * * *$} \\
\hline & $\mathrm{C}$ & 25.9 & 6.0 & \\
\hline \multirow{2}{*}{ Originalidad Narrativa } & $\mathrm{E}$ & 63.8 & 7.0 & \multirow{2}{*}{$.005 * *$} \\
\hline & $\mathrm{C}$ & 35.2 & 6.6 & \\
\hline \multirow{2}{*}{ Creatividad Gráfica } & $\mathrm{E}$ & 84.0 & 4.9 & \multirow{2}{*}{$.001 * * *$} \\
\hline & $\mathrm{C}$ & 60.0 & 4.6 & \\
\hline \multirow{2}{*}{ Originalidad Gráfica } & $\mathrm{E}$ & 69.6 & 5.4 & \multirow{2}{*}{$.042 *$} \\
\hline & $\mathrm{C}$ & 55.0 & 5.0 & \\
\hline \multirow{2}{*}{ Elaboración } & $\mathrm{E}$ & 76.4 & 6.4 & \multirow{2}{*}{$.002 * *$} \\
\hline & $\mathrm{C}$ & 46.9 & 6.0 & \\
\hline \multirow{2}{*}{ Detalles Especiales } & $\mathrm{E}$ & 94.4 & 4.7 & \multirow{2}{*}{$.0001 * * *$} \\
\hline & $\mathrm{C}$ & 76.0 & 4.4 & \\
\hline \multirow{2}{*}{ Título } & $\mathrm{E}$ & 41.2 & 5.8 & \multirow{2}{*}{.128} \\
\hline & $\mathrm{C}$ & 31.5 & 5.4 & \\
\hline \multirow{2}{*}{ Creatividad General } & $\mathrm{E}$ & 65.4 & 7.0 & \multirow{2}{*}{$.005 * *$} \\
\hline & $\mathrm{C}$ & 37.3 & 6.6 & \\
\hline
\end{tabular}

Los resultados obtenidos de la creatividad narrativa y gráfica en la prueba de seguimiento a nivel nomotético muestran diferencias significativas entre el GE y el GC en la mayoría de las variables de la creatividad (Tabla 3). Concretamente, el desarrollo de la creatividad del GE es significativamente mayor que el del GC en la flexibilidad narrativa, los detalles especiales y la creatividad gráfica $(p<.001)$; en la elaboración, la creatividad narrativa, la creatividad gráfica y en la creatividad general $(p<.01)$ y; en la originalidad gráfica $(p<.05)$. El desarrollo de la fluidez narrativa y el título del GE es mayor que el del GC, pero la diferencia entre uno y otro no es significativa $(p>.05)$. 
Según lo expuesto, se puede afirmar que las diferencias en el desarrollo de la creatividad entre un grupo y otro se mantienen estables transcurridos tres meses a la implementación del programa en todas las variables objeto de estudio, excepto para la fluidez narrativa que las diferencias existentes entre un grupo y otro no son significativas o aumentan a favor del GE, como observamos en la originalidad gráfica.

Por otro lado, los resultados obtenidos a nivel ideográfico confirman los resultados obtenidos a nivel nomotético. En la Tabla 4 se presenta la proporción de participantes que han desarrollado la creatividad de un modo significativo en ambos grupos de estudio, así como las diferencias observadas entre un grupo y otro. Según estos resultados, el GE ha obtenido un desarrollo significativo en la creatividad figurativa del $90 \%$ y en la creatividad verbal del $62 \%$. Además, un porcentaje elevado de participantes del GE han desarrollado de forma significativa las variables de la creatividad verbal (fluidez: 57\%; flexibilidad: 81\%; originalidad: 57\%) como de la creatividad figurativa (fluidez: 76\%; flexibilidad: 52\%; originalidad: $86 \%$; elaboración: 76\%). Finalmente, la diferencia en el desarrollo de la creatividad verbal y figurativa entre un grupo y otro es del $37 \%$ y del $36 \%$ respectivamente a favor del GE.

Tabla 4. Proporción del desarrollo significativo de la creatividad entre la prueba inicial y final en los grupos de estudio y su diferencia entre ambos grupos de estudio.

\begin{tabular}{ccccc}
\hline \multirow{2}{*}{ Variable } & $\begin{array}{c}\text { Grupo Expe- } \\
\text { rimental (GE) }\end{array}$ & $\begin{array}{c}\text { Grupo Con- } \\
\text { trol (GC) }\end{array}$ & Diferencia GE-GC \\
\hline \multirow{2}{*}{$\begin{array}{c}\text { Creatividad } \\
\text { Verbal }\end{array}$} & Fluidez & $57 \%$ & $21 \%$ & $36 \%$ \\
& Flexibilidad & $81 \%$ & $33 \%$ & $48 \%$ \\
& Criginalidad & $57 \%$ & $29 \%$ & $28 \%$ \\
\multirow{2}{*}{ Creatividad Ver- } & $\mathbf{6 2 \%}$ & $\mathbf{2 5 \%}$ & $\mathbf{3 7 \%}$ \\
& Fluidez & $76 \%$ & $71 \%$ & $5 \%$ \\
& Flexibilidad & $52 \%$ & $33 \%$ & $19 \%$ \\
& Originalidad & $86 \%$ & $71 \%$ & $15 \%$ \\
& Elaboración & $\mathbf{7 6 \%}$ & $21 \%$ & $55 \%$ \\
& Creatividad Figu- & $\mathbf{9 0 \%}$ & $\mathbf{5 4 \%}$ & $\mathbf{3 6 \%}$ \\
\hline
\end{tabular}

\section{Conclusiones}

Los resultados obtenidos en este estudio ponen de manifiesto la importancia del desarrollo de la creatividad en la educación secundaria y en el currículum a través de los objetivos y materias del mismo y de los procesos de enseñanza-aprendizaje utilizados por el profesora- 
do en el aula. De este modo, los participantes del GE han desarrollado de forma significativa la creatividad a nivel intragrupal como intergrupal.or Por lo tanto, la intervención resulta positiva y el programa ha logrado los efectos que pretendía, que era el desarrollo de las creatividad de un grupo de estudiantes de segundo curso de ESO.

Los resultados de esta investigación coinciden con los observados en estudios precedentes dentro del ámbito del desarrollo de la creatividad realizados por diversos autores tanto a nivel nacional (Allueva, 2002; Garaigordobil y Berrueco, 2011; López, 2001; Navarro, 2008; Prieto et al., 2002), Europeo (European Union, 2006), como internacional (Crutchfield y Davis, 1966, De Bono, 1973, 1985; De Sánchez, 1996; Harvard University, 2010) que han demostrado un desarrollo significativo de la creatividad en distintas etapas y niveles educativos.

Además, los efectos del programa en el desarrollo de la creatividad verbal y figurativa obtenidos en las pruebas de seguimiento, se mantienen en ambas variables $(p<.05)$ una vez finalizado el programa y transcurridos tres meses después del mismo. Una vez finalizado el programa se observa un desarrollo significativo de la creatividad verbal y en todas sus variables y un desarrollo significativo de la creatividad gráfica y de la elaboración. Transcurridos tres meses después de la intervención, se observa un desarrollo significativo de la creatividad en las mismas variables excepto en la fluidez verbal, que su desarrollo deja de ser significativo $(p>.05)$ y en la originalidad gráfica $(p<.05)$ que su desarrollo pasa a ser significativo. Este mantenimiento de los resultados en la prueba de seguimiento y la concordancia en el análisis realizado a nivel nomotético e ideográfico, muestra una elevada fiabilidad del programa de intervención.

No obstante, el estudio presenta algunas limitaciones, tales como que el desarrollo de la creatividad figurativa o gráfica no ha sido tan elevado como hubiese sido deseado. Este factor se puede deber a una escasez de actividades dentro del programa orientadas al desarrollo de la creatividad figurativa o gráfica, lo que demuestra que este factor puede haberse desarrollado por igual en ambos grupos o que su incidencia no ha sido muy elevada en el GE. Por lo tanto, en posteriores intervenciones, será conveniente aumentar el número de actividades relacionadas con la creatividad figurativa o gráfica. 
Otra de las posibles sugerencias que cabe añadir a la mejora del estudio está implícita desde el punto de vista del diseño. En primer lugar, los resultados deben ser asumidos con cautela ya que representan a una muestra pequeña de estudiantes, la cual debería ampliarse en futuras intervenciones. Por otro lado, desde un punto de vista evaluativo del constructo creativo, se ha demostrado que los procesos de pensamiento divergente describen la esencia de la creatividad pero no es reductible a éstos (Santaella, 2006; Sternberg \& Kaufman, 2010). En este estudio se han evaluado eminentemente dichos procesos sin profundizar en otros factores como la influencia del contexto (sociales, educativa, ambientales y familiares), la personalidad (iniciativa, sensibilidad, autoestima, independencia, entre otros) o el producto creativo (innovación en el resultado, objetos y productos). Por lo que quedaría abierta una posible línea de investigación para futuras investigaciones.

Sin embargo, a pesar de estas limitaciones, se puede afirmar que gracias a este estudio se ha demostrado que las habilidades creativas se pueden potenciar y desarrollar de forma eficaz si se realizan los esfuerzos y actividades pertinentes mediante el uso de una metodología apropiada.

Debemos tener en cuenta el contexto social en el que está inmersa la intervención ya que, en muchos casos, no se puede apreciar el desarrollo de la creatividad en el currículum debido al estricto seguimiento de los programas educativos y a la poca flexibilidad de los mismos para implementar una metodología innovadora orientada al desarrollo de la creatividad (Santaella, 2006). Para desarrollar la creatividad en el contexto educativo, debe fomentarse la formación del profesorado en este tipo de habilidades, estrategias y metodologías con el fin de que el alumnado pueda adquirir nuevas competencias, más acordes a las necesidades planteadas en el contexto social del siglo XXI.

\section{Referencias}

Allueva, P. (2002). Desarrollo de la creatividad: Diseño y evaluación de un programa de intervención, Revista Persona, 5, 67-81. Lima: Universidad de Lima.

Allueva, P. (2007). Habilidades del Pensamiento. En M. Liesa, P. Allueva y M. Puyuelo (Coords.), Educación y acceso a la vida adulta de Personas con Discapacidad (pp. 133158). Barbastro, Huesca: Fundación R.J. Sender. 
Amabile, T. M. (1983). The social psychology of creativity: a componencial conceptualization. Journal of Personality and Social Psychology, 45 (2), 357-376.

Arlin, P. K. (1975). Cognitive development in adulthood: a fifth stage? Developmental Psychology, Vol.11, 5, pp. 602-606. Abstract. Retrieved the $10^{\text {th }}$ of may of 2011 from, http://www.eric.ed.gov/ERICWebPortal/search/detailmini.jsp?_nfpb=true\&_\&ERICExt Search_SearchValue_0=EJ129933\&ERICExtSearch_SearchType_0=no\&accno=EJ1299 33.

Artola, T., Barraca, J., Martín, C., Mosteiro, P., Ancillo, I. y Poveda, B. (2008). Prueba de Imaginación Creativa para Jóvenes. Madrid: TEA.

BernabeU, N. y Goldstein, A. (2009). Creatividad y aprendizaje. El juego como herramienta pedagógica. Buenos Aires: Narcea.

Education, Audiovisual y Culture Executive Agency (2008). Creative Learning Communities. Progress report. Brussels: Education, Audiovisual \& Culture Executive Agency. Retrieved the 10th of 2012 from http://www.adameurope.eu/prj/6744/prj/2010_3558_PR_CLC_pub.pdf.

European Union (2010). Creative Learning Communities. Projects and products for Leonardo da Vinci. Retrieved the 2nd of april of 2012 from http://www.adameurope.eu/adam/project/view.htm?prj=6744.

European Union (2008). Decision No 1350/2008/EC of the European Parliament and of the Council of 16 December 2008 concerning the European Year of Creativity and Innovation. Strasbourg, France: Official Journal of the European Journal. Retrieved the $10^{\text {th }}$ of January of 2011 from:

http://eurlex.europa.eu/LexUriServ/LexUriServ.do?uri=OJ:L:2008:348:0115:0117:EN:P DF.

European Union (2006). Recommendation of the European Parliament and of the Council of 18 December 2006 of key competences for lifelong learning (2006/962/EC). Brussels: Official Journal of the European Union. Retrieved the 8th of september of 2011 fromhttp://eur-

lex.europa.eu/LexUriServ/LexUriServ.do?uri=OJ:L:2006:394:0010:0018:en:PDF.

European Union (2005). Proyecto europeo para la ideación y validación de una innovadora metodología formativa. Milan: Paoline.

European University Association (2007). Creativity in Higher Education. Report on EUA Creativity Project. Brussels: European University Association. Retrieved the $10^{\text {th }}$ of January of 2011 from http://www.eua.be. 
Coolican, H. (2009). Research methods and Statistics in Psychology: London: Hodder Education.

Covington, M.V., Crutchfield, R.S. y Davis, L. (1966). The productive thinking program. Berkcley C.A.: Brazelton.

De Bono, E. (1970). Lateral thinking: creativity step by step. New York: Harper \& Row.

De Bono, E. (1973). CoRT thinking. Blanford, England: Direct Educational Services.

De Bono, E. (1985). The CoRT Thinking Program. En S. F. Chipman, J. W. Segal \& R. Glaser (Eds.), Thinking and learning skills. Relating instruction to research, Vol. 1 (pp. 363-388). Hillsdale, NJ: Erlbaum.

Ferrando, M., Ferrándiz, C., Rosario, M., Sánchez, C., Parra, J., y Prieto, M. D. (2007). Estructura interna y baremación del Test de Pensamiento Creativo de Torrance. Psicothema, 19, 489-496.

Garaigordobil, M. y Berrueco, L. (2011). Effects of play program on creative thinking of preschool children. Spanish Journal of Psychology, 14(2), 608-618.

Gardner, H. (1993). Creativity minds: an anatomy of creativity. New York: Perseus Books Group.

Gardner, H. (2006). Five minds for the future. Boston: Harvard Business School Press.

Guilford, J. P. (1950). Creativity. American Psychologist, 5, 444-454.

Guilford, J. P. (1967a). The nature of human Intelligence. New York: McGraw Hill

Guilford, J. P. (1967b). Factors that aid and hinder creativity. En J. Curtis, G. Demos \& E. P. Torrrance (Eds.), Creativity: its educational implications. New York: Wiley.

Harvard University (2010). Proyect Zero. July 2010. Cambridge: Harvard Graduate School of Education. Retrieved the 10th of January of 2011 from http://www.pz.harvard.edu/research/ProjectZeroCurrentWork_July\%202010.pdf.

Larraz, N. (2011). Desarrollo de las habilidades creativas y metacognitivas en la Educación Secundaria Obligatoria. Programa de intervención. Tesis Doctoral sin publicar. Zaragoza: Departamento de Psicología y Sociología; Universidad de Zaragoza.

Lipman, M. (1991). Thinking in education. New York: Cambridge University Press.

López, O. (2001). Evaluación y desarrollo de la creatividad. Tesis Doctoral. Murcia: Departamento de métodos de investigación y diagnóstico en educación. Universidad de Murcia.

Lorna, P. M. (1998). Inventory of barriers to creative thought and innovative action, Vol. 8 (pp. 46-53). The Pfeiffer Library (2nd Ed.). 
Mayer, R. E. (1992). Thinking, problem solving and cognition (2nd Ed.). New York: Freeman.

Navarro, J. (2008). Mejora de la creatividad en el aula de primaria. Tesis Doctoral. Departamento de Personalidad, Evaluación y Tratamiento psicológicos. Murcia: Universidad de Murcia.

Nickerson, R. S., Perkins, D. N. \& Smith, E. E. (1985). The teaching of the thinking. New York: Lawrence and Erlbaum.

Osborn, A. F. (1953). Applied imagination. Principles and procedures of creative thinking. New York: Scribner's Sons.

Perkins, D. N. (1993) La creatividad y su desarrollo: una aproximación disposicional. En J. Beltrán, V. Bermejo y D. Prieto (Eds.), Intervención Psicopedagógica (pp. 91-107). Madrid: Pirámide.

Prieto, M. D., López, O., Bermejo, M. R., Renzulli, J. y Castejón, J. L. (2002). Evaluación de un programa de desarrollo de la creatividad. Psicothema, 14 (2), 410-414.

Recio, H. (2005). Creatividad en la solución de problemas. México: Trillas.

Rodari, G. (2004). Gramática de la fantasía. Introducción al arte de contar historias. Barcelona: Planeta.

Runco, M. A. (2007). Creativity theories and themes: research, development and practice. Burlington, MA: Elsevier.

Runco, M. A. (2010). Divergent thinking, creativity, and ideation. In J.C. Kaufman \& R. J. Sternberg (Eds.), The Cambridge handbook of creativity (pp. 413- 446). New York: Cambridge University Press.

Santaella, M. (2006). La evaluación de la creatividad. Sapiens, 7 (2), 89-106. Recuperado el 7 de noviembre de 2012 de http://redalyc.uaemex.mx/

Sampascual, G. (2007). Psicología de la educación, Vol 2. Madrid: UNED.

Sánchez de, M. (1996). Programa para el desarrollo de las habilidades del pensamiento: creatividad. México: Trillas.

Simberg, A. L. (1971). Training creative thinking. New York: Rinehart and Winston.

Sternberg, R. J. (1985). Beyond IQ: A Triarchic Theory of Intelligence. Cambridge: Cambridge University Press.

Sternberg, R. J. y Lubart, T.I. (1995). Defying the crowd: Cultivating creativity in a culture of conformity. New York: Free Press. 
Sternberg, R. J., y Kaufman, J.C. (2010). Constraints on creativity. In J. C. Kaufman \& R. J. Sternberg (Eds.), The Cambridge handbook of creativity (pp. 467- 482). New York: Cambridge University Press.

Torrance, E. P. (1966). Torrance Test of Creative Thinking (TTCT). Norms Technical Manual. Princenton, NJ: Personal Press.

Torre, S. de la (2006). Creatividad en la educación. En S. de la Torre y V. Violant (Coords. y Dir.), Comprender y evaluar la creatividad, Vol. 1 (pp.-309-322). Málaga: Aljibe.

Unesco (2011). International Standard Classification of Education. UNESCO: Paris.

Woolfolk, A. (2010). Educational psychology (11th Ed.). Columbus, OH: Pearson/Allyn \& Bacon. 